\title{
Development of a Web-Based System for Exploring Cancer Risk With Long-term Use of Drugs: Logistic Regression Approach
}

Hsuan-Chia Yang ${ }^{1,2,3}$, PhD; Md Mohaimenul Islam ${ }^{1,2,3}$, PhD; Phung Anh Alex Nguyen ${ }^{2}$, PhD; Ching-Huan Wang ${ }^{1}$, MSc; Tahmina Nasrin Poly ${ }^{1,2,3}$, MSc; Chih-Wei Huang ${ }^{2}, \mathrm{PhD}$; Yu-Chuan Jack Li ${ }^{1,2,3,4,5}, \mathrm{MD}, \mathrm{PhD}$

${ }^{1}$ Graduate Institute of Biomedical Informatics, College of Medical Science and Technology, Taipei Medical University, Taipei, Taiwan

${ }^{2}$ International Center for Health Information Technology, Taipei Medical University, Taipei, Taiwan

${ }^{3}$ Research Center of Big Data and Meta-analysis, Wan Fang Hospital, Taipei Medical University, Taipei, Taiwan

${ }^{4}$ Department of Dermatology, Wan Fang Hospital, Taipei Medical University, Taipei, Taiwan

${ }^{5}$ TMU Research Center of Cancer Translational Medicine, Taipei Medical University, Taipei, Taiwan

\section{Corresponding Author:}

Yu-Chuan Jack Li, MD, PhD

Graduate Institute of Biomedical Informatics

College of Medical Science and Technology

Taipei Medical University

15 F, No. 172-1, Sec 2, Kellung Rd

Da'an District

Taipei, 110

Taiwan

Phone: 886227361661 ext 7600

Email: jaak88@gmail.com

\section{Abstract}

Background: Existing epidemiological evidence regarding the association between the long-term use of drugs and cancer risk remains controversial.

Objective: We aimed to have a comprehensive view of the cancer risk of the long-term use of drugs.

Methods: A nationwide population-based, nested, case-control study was conducted within the National Health Insurance Research Database sample cohort of 1999 to 2013 in Taiwan. We identified cases in adults aged 20 years and older who were receiving treatment for at least two months before the index date. We randomly selected control patients from the patients without a cancer diagnosis during the 15 years (1999-2013) of the study period. Case and control patients were matched 1:4 based on age, sex, and visit date. Conditional logistic regression was used to estimate the association between drug exposure and cancer risk by adjusting potential confounders such as drugs and comorbidities.

Results: There were 79,245 cancer cases and 316,980 matched controls included in this study. Of the 45,368 associations, there were $2419,1302,662$, and 366 associations found statistically significant at a level of $P<.05, P<.01, P<.001$, and $P<.0001$, respectively. Benzodiazepine derivatives were associated with an increased risk of brain cancer (adjusted odds ratio [AOR] 1.379, 95\% CI 1.138-1.670; $P=.001$ ). Statins were associated with a reduced risk of liver cancer (AOR 0.470, 95\% CI 0.426-0.517; $P<.0001$ ) and gastric cancer (AOR 0.781, 95\% CI 0.678-0.900; $P<.001$ ). Our web-based system, which collected comprehensive data of associations, contained 2 domains: (1) the drug and cancer association page and (2) the overview page.

Conclusions: Our web-based system provides an overview of comprehensive quantified data of drug-cancer associations. With all the quantified data visualized, the system is expected to facilitate further research on cancer risk and prevention, potentially serving as a stepping-stone to consulting and exploring associations between the long-term use of drugs and cancer risk.

(JMIR Public Health Surveill 2021;7(2):e21401) doi: 10.2196/21401

\section{KEYWORDS}

cancer; risk; prevention; chemoprevention; long-term-use drugs; drug; epidemiology; temporal model; modeling; web-based system 


\section{Introduction}

In recent decades, the prevalence of chronic medical conditions such as arthritis, osteoporosis, diabetes, hypertension, and cardiovascular disease has increased [1]. Patients with multimorbidity are more likely to have multidrug treatments; it is, in fact, common in older populations. Patients also may need a longer duration of treatment to get rid of these conditions, which leaves these patients vulnerable to unwanted side effects [2-4]. Multiple studies have already reported that long-term use of drugs has been increasing tremendously $[5,6]$. Therefore, a growing concern regarding the safety issues associated with long-term prescriptions has recently attracted widespread media attention. Physicians are often asked about the appropriateness of long-term therapy for specific patients.

There has been a significant rise in the number of published studies in which commonly used medications were found to increase or decrease the risk of cancer [7]. Long-term use of insulin seems to be associated with an increased risk of pancreas, liver, kidney, and stomach cancers [8], whereas a protective association was observed between metformin use and colorectal cancer risk in patients with diabetes mellitus [9]. Additionally, antihypertensive drugs are associated with an increased risk of skin cancer [10], but they might have a possible beneficial effect on breast cancer risk [11]. However, there has been substantial controversy about these studies' validity, and investigations with varying study designs and populations have often arrived at different conclusions. A prudent decision is immediately needed in clinical practices because the use of commonly prescribed medications has been increasing, and the resulting burden of cancer can be substantial at the population level [12].

Big data approaches seem to offer an immense opportunity to generate strong evidence for taking insightful clinical and public health action [13]. Data from electronic medical records and other extended patient registries have been offering expanded research power, especially for analytic studies aiming at association, classification, and prediction [14]. Epidemiological studies have already established a temporal relationship between drugs and diseases and have evaluated a wide range of outcomes. Clinical knowledge is constantly developing as new drug-disease discoveries are made and practices are changed. The knowledge of these associations is valuable but often buried in texts within a range of published literature. Through a web-based approach, information associated with drugs and disease risk can be a great source to understanding the magnitude of the risk between them. The availability of a wide range of associations may be valuable for a variety of applications, including clinical decision support (eg, treatment recommendation), information retrieval, and data mining. We therefore developed a web-based system focusing on the long-term use of commonly prescribed medications, including antihypertensives, antihyperlipidemics, antidiabetics, antihyperuricemics, anxiolytics, hypnotics, sedatives, and nonsteroidal anti-inflammatory drugs (NSAIDs) and 18 different kinds of cancer risk. We developed a system sorted by age, gender, and duration of therapy for exploring cancer risk with commonly used medications.

\section{Methods}

\section{Ethical Standard}

This study is part of a larger project aimed at assessing the effect of most common medications on 20 cancer sites using a population-based nested case-control design. The National Health Insurance Research Database (NHIRD) safeguards the privacy and confidentiality of all beneficiaries and transfers health insurance data to health researchers after ethical approval has been obtained. In this analysis, access to the NHIRD was approved by the Taipei Medical University Joint Institutional Review Board.

\section{Setting and Data Source}

This case-control study was carried out using records from the Taiwan NHIRD, which was established in 1995 and has collects all claims of beneficiaries under the National Health Insurance (NHI) program. The program covers more than $99 \%$ of the total population (a total of $23,430,000$ ) and has contracted with $97 \%$ of the hospitals and clinics in Taiwan. The NHIRD comprised claims data of 2,000,000 individuals randomly selected from all insured enrollees. This sample represents the original medical claims for all residents in Taiwan covered under the NHI program. The database included specific data on medications prescribed, laboratory and diagnostic test data, dates of visits, lengths of hospitalization, and diagnoses. Diagnoses were coded according to the International Classification of Disease, Ninth Revision, Clinical Modification (ICD-9-CM). Drugs were coded based on the World Health Organization Anatomical Therapeutic Chemical (ATC) classifications (WHO Collaborating Centre for Drug Statistics Methodology ATC/DDD Index). The database used in this study can be interlinked by the scrambled, unique, individual personal identification number.

\section{Case and Control Selection Criteria}

We identified cases in adults who were aged 20 years or older and had received treatment at least two months before the index date. The index date was defined as the date of a cancer diagnosis. We used the ICD-9-CM to identify patients with cancer as cases. Among the NHIRD cases, eligibility criteria for case patients were (1) registration as patients with cancer in the catastrophic illness file, (2) diagnosis of primary cancer in inpatient admission, (3) treatment with any cancer drug from outpatient visits or inpatient admission, (4) a cancer-specific procedure from outpatient visits or inpatient admission, and (5) more than 4 cancer-specific examinations or more than 1 cancer-related procedure (radiotherapy, chemotherapy, or treatment tracking of cancer) from outpatient visits or inpatient admission.

We randomly selected control patients from patients without a cancer diagnosis during the 15 years (1999-2013) of the study period. Case and control patients were matched 1:4 based on age, sex, and visit date (Figure 1). 
Figure 1. Workflow of the case-control study design. NHI: National Health Insurance.

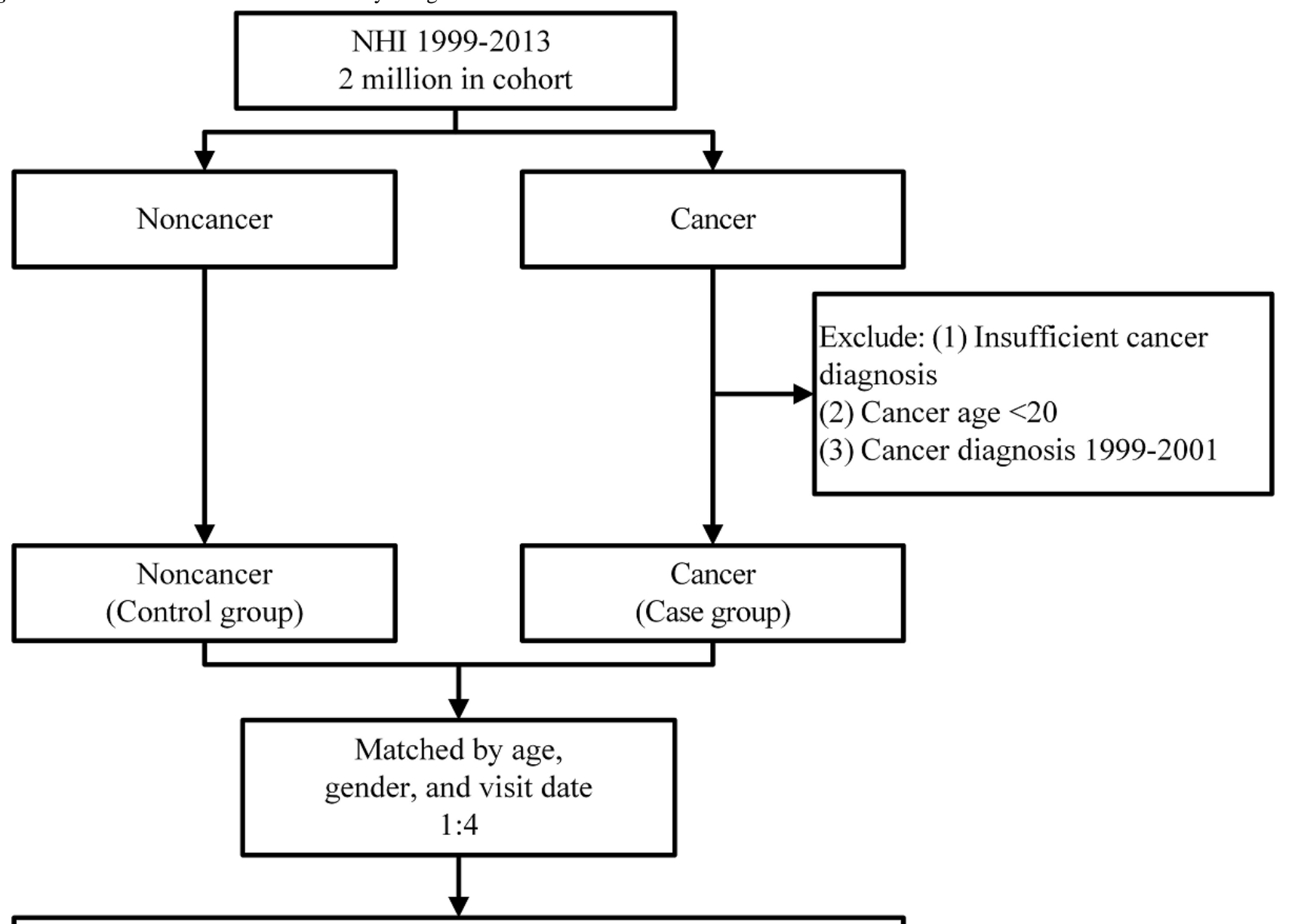

Explore the association between the drugs and cancer risk (conditional logistic regression)

\section{Primary Outcomes}

We quantified the risks of common cancers in Taiwan, comparing patients treated with (1) antihypertensives; (2) antihyperlipidemics; (3) antidiabetics; (4) antihyperuricemics; (5) anxiolytics, hypnotics, and sedatives; or (6) NSAIDs against those not prescribed any of these medications. We investigated the following cancers using their corresponding ICD-9-CM codes: oral cancer (140-149.xx, excluding 142.xx and 147.xx), esophageal cancer (150.xx), gastric cancer (151.xx), colorectal cancer (153.xx, rectum 154.xx), liver cancer (155.xx), pancreatic cancer (157.xx), lung cancer (162.xx), skin cancer (172-173.xx), female breast cancer (174.xx), cervical cancer (180.xx), endometrial cancer (182.xx), ovarian cancer (183.xx), prostate cancer (185.xx), bladder cancer (188.xx), kidney cancer (189.xx), brain cancer (191.xx), thyroid cancer (193.xx),
non-Hodgkin disease (200.xx, 202.xx, 203.xx), leukemia (204-208.xx), and all cancers (140-208.xx).

\section{Use of Drugs}

We defined the index date as the date of a cancer diagnosis. The drug exposure was analyzed only before the index date, and we defined drug users as those who filled prescriptions of at least 60 days during admissions and outpatient visits within the 2 years before the index date (Figure 2). This definition was considered for 6 long-term drug groups, namely (1) antihypertensives; (2) antihyperlipidemics; (3) antidiabetics; (4) antihyperuricemics; (5) antianxiety agents, hypnotics, and sedatives; and (6) NSAIDs. Those having no exposure to or receiving these drugs for less than two months were classified as nondrug users. 
Figure 2. Drugs exposure.

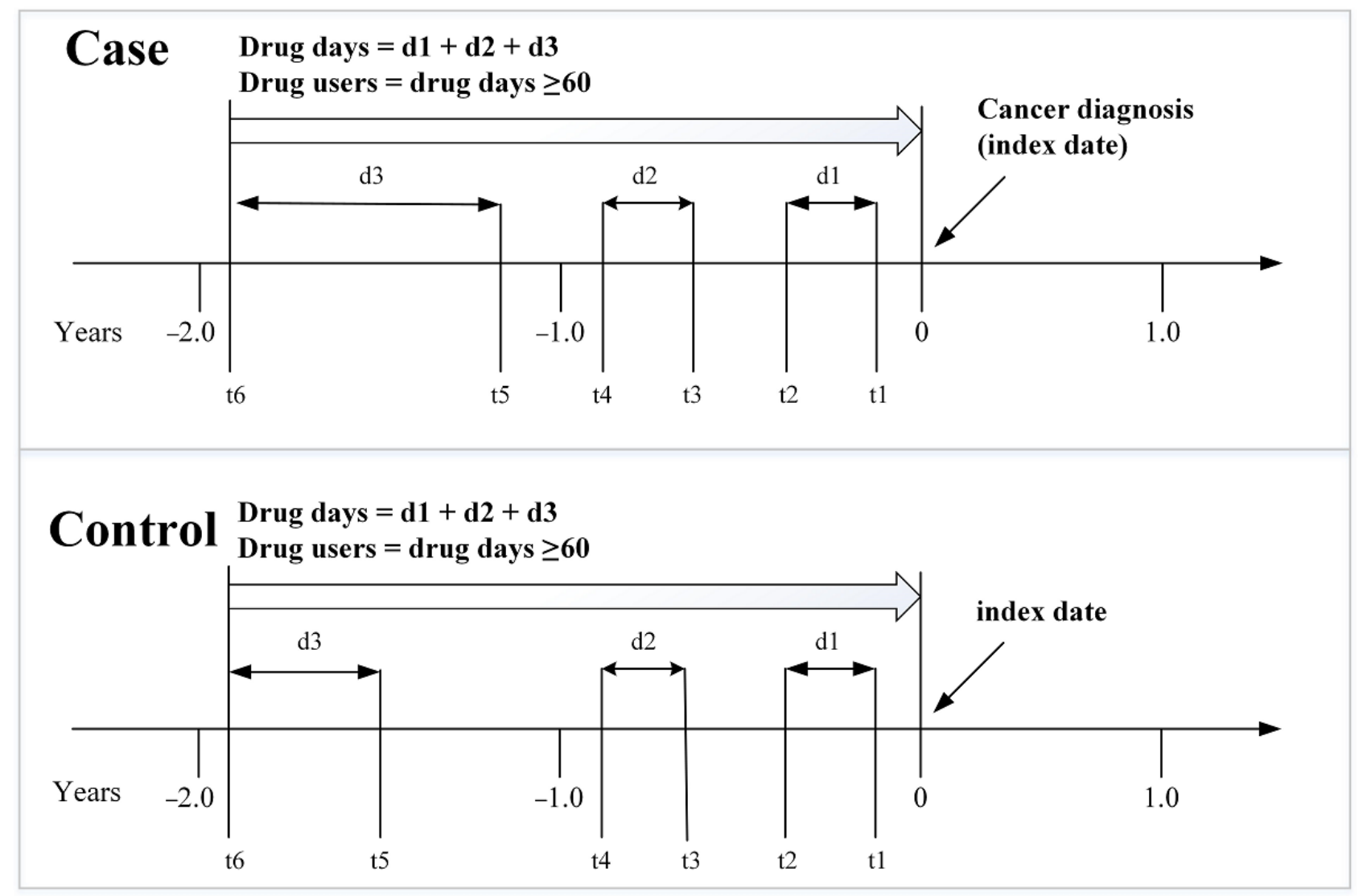

\section{Potential Confounders}

Comorbidities and medications identified as confounders were adjusted in this study. Comorbidities were defined using the Charlson Comorbidity Index [15], retrieved from outpatient visits before the index date. Any of the comorbidities as confounders were required to be diagnosed at least twice within the 2 years ( 720 days) before the index date, except for chronic pulmonary disease, which required a diagnosis made more than 4 times. In addition, aspirin (ATC: B01AC06), statins (ATC: C10AA), and metformin (ATC: A10BA2) were also confounders because they might potentially influence the risk of cancers. Exposure to a confounding drug was defined as treatment with the drug for at least 60 days during the 2 years before the index date.

\section{Statistical Analysis}

The McNamara test and paired $t$ test were used to test the difference between the case and control groups [16]. Next, conditional logistic regression was conducted to estimate the association between drug exposure and cancer risk by adjusting potential confounders [17]. Table 1 shows our study variables, and conditional logistic regression (temporal model) was adopted to investigate the association between the long-term use of drugs and cancer risk. Age was divided into 4 categories: 20 to 39 years, 40 to 64 years, $\geq 65$ years, and $\geq 20$ years. Gender was classified as male, female, and both. The basic equation of the model was as below, and it may have been slightly modified in different study drug groups. 
Table 1. Study variables.

\begin{tabular}{|c|c|c|c|c|}
\hline Variables & Type & Descriptive & Statistical model & Definition \\
\hline ID & Nominal & $\mathrm{N} / \mathrm{A}^{\mathrm{a}}$ & No & N/A \\
\hline Age & Ordinal & N/A & No & N/A \\
\hline Sex & Binomial & 1: Yes, 0: No & No & N/A \\
\hline Study drug (exposure) & Binomial & 1: Yes, 0: No & Yes & Independent \\
\hline Cancer (outcome) & Binomial & 1: Yes, 0: No & Yes & Dependent \\
\hline Myocardial infarction & Binomial & 1: Yes, 0: No & Yes & Confounding \\
\hline Congestive heart failure & Binomial & 1: Yes, 0: No & Yes & Confounding \\
\hline Peripheral vascular disease & Binomial & 1: Yes, 0: No & Yes & Confounding \\
\hline Cerebrovascular disease & Binomial & 1: Yes, 0: No & Yes & Confounding \\
\hline Dementia & Binomial & 1: Yes, 0: No & Yes & Confounding \\
\hline Chronic pulmonary disease & Binomial & 1: Yes, 0: No & Yes & Confounding \\
\hline Rheumatic disease & Binomial & 1: Yes, 0: No & Yes & Confounding \\
\hline Peptic ulcer disease & Binomial & 1: Yes, 0: No & Yes & Confounding \\
\hline $\begin{array}{l}\text { Liver disease (mild, moderate, and se- } \\
\text { vere) }\end{array}$ & Binomial & 1: Yes, 0: No & Yes & Confounding \\
\hline $\begin{array}{l}\text { Diabetes (with or without chronic } \\
\text { complication) }\end{array}$ & Binomial & 1: Yes, 0: No & Yes & Confounding \\
\hline Hemiplegia or paraplegia & Binomial & 1: Yes, 0: No & Yes & Confounding \\
\hline Renal disease & Binomial & 1: Yes, 0: No & Yes & Confounding \\
\hline $\mathrm{CCI}^{\mathrm{b}}$ scores & Ordinal & N/A & Yes & Confounding \\
\hline Metformin & Binomial & 1: Yes, 0: No & Yes & Confounding \\
\hline Aspirin & Binomial & 1: Yes, 0: No & Yes & Confounding \\
\hline Statin & Binomial & 1: Yes, 0: No & Yes & Confounding \\
\hline Matching number (case match control) & Nominal & N/A & Yes & Stratified \\
\hline
\end{tabular}

${ }^{\mathrm{a}} \mathrm{N} / \mathrm{A}$ : not applicable.

${ }^{\mathrm{b}}$ Charlson Comorbidity Index.

Data analysis and results were performed using SAS software (version 9.4; SAS Institute) [18]. The results were expressed in adjusted odds ratios (AORs), which is $\mathrm{e}^{\beta 1}$ with different confidence intervals, like $95 \%, 99 \%$, and $99.9 \%$. All statistical tests were 2-sided.

\section{Web-Based System}

After analyzing the associations between the long-term use of drugs and cancer risk, we built a web-based system to include all associations [19]. The back end of this web-based system includes a server, database, and application. We used Apache (Apache Software Foundation), MySQL (Oracle Corporation), and PHP (Hypertext Preprocessor) framework for developing the server. Apache is the most commonly used web server software and supports a variety of compiled modules. MySQL is a relational database management system, and PHP is a programming language designed primarily for web development. In this study, we inputted the associations into the database (MySQL) and used PHP to access and process the database. The web-based interface was designed accordingly with HTML, CSS, Bootstrap, JavaScript, and React.

\section{Results}

\section{Baseline Characteristics}

We identified 79,245 participants newly diagnosed with cancer between 2002 and 2013 from 2 million people (Table 2). After matching each case with 4 controls, we included 316,980 matched control patients in this study. The mean age was 59.2 years for both the case and control groups. A slight majority of the participants were male $(201,295 / 396,225,50.80 \%)$, and most participants $(202,544 / 396,225,51.12 \%)$ were aged 40 to 64 years. The prevalence of peptic ulcer disease $(12,760 / 79,245$, $16.10 \%$ vs $34,283 / 316,980,10.82 \%$ ) and liver disease $(11,671 / 79,245,14.73 \%$ vs $20,647 / 316,980,6.51 \%)$ in the case group was higher than that in the control group. After associations between long-term use of drugs and cancer risk were comprehensively analyzed and stratified by age and sex, we obtained 45,368 associations in total, of which 2419, 1302, 662, and 366 associations were found statistically significant at a level of $P<.05, P<.01, P<.001$, and $P<.0001$, respectively (Table S1 in Multimedia Appendix 1). 
Table 2. Baseline characteristics of case patients and control patients.

\begin{tabular}{|c|c|c|c|}
\hline Characteristics & Case patients (with cancer) $(\mathrm{n}=79,245)$ & $\begin{array}{l}\text { Control patients (without cancer) } \\
(\mathrm{n}=316,980)\end{array}$ & $P$ value \\
\hline \multicolumn{4}{|l|}{ Age (years) } \\
\hline Age (years), mean (SD) & $59.20(15.23)$ & $59.21(15.24)$ & $\mathrm{N} / \mathrm{A}^{\mathrm{a}}$ \\
\hline $20-39, \mathrm{n}(\%)$ & $8,292(10.5)$ & $33,168(10.5)$ & N/A \\
\hline $40-64, \mathrm{n}(\%)$ & $40,504(51.1)$ & $162,040(51.1)$ & N/A \\
\hline$\geq 65, \mathrm{n}(\%)$ & $30,449(38.4)$ & $121,772(38.4)$ & N/A \\
\hline \multicolumn{4}{|l|}{ Gender, n (\%) } \\
\hline Male & $40,259(50.8)$ & $161,036(50.8)$ & N/A \\
\hline Female & $38,986(49.2)$ & $155,944(49.2)$ & N/A \\
\hline \multicolumn{4}{|l|}{ Comorbid conditions, n (\%) } \\
\hline Myocardial infarction & $372(0.47)$ & $1729(0.55)$ & $<.0001$ \\
\hline Congestive heart failure & $2557(3.23)$ & $9344(2.95)$ & $<.0001$ \\
\hline Peripheral vascular disease & $1143(1.44)$ & $4187(1.32)$ & $<.0001$ \\
\hline Cerebrovascular disease & $5306(6.70)$ & $22,609(7.13)$ & $<.0001$ \\
\hline Dementia & $1205(1.52)$ & $5522(1.74)$ & $<.0001$ \\
\hline Chronic pulmonary disease & $5951(7.51)$ & $20,423(6.44)$ & $<.0001$ \\
\hline Rheumatic disease & $1138(1.44)$ & $3901(1.23)$ & $<.0001$ \\
\hline Peptic ulcer disease & $12,760(16.10)$ & $34,283(10.82)$ & $<.0001$ \\
\hline Liver disease & $11,671(14.73)$ & $20,647(6.51)$ & $<.0001$ \\
\hline Diabetes & $9143(11.54)$ & $32,532(10.26)$ & $<.0001$ \\
\hline Hemiplegia or paraplegia & $490(0.62)$ & $2300(0.73)$ & $<.0001$ \\
\hline Renal disease & $2793(3.52)$ & $7453(2.35)$ & $<.0001$ \\
\hline \multicolumn{4}{|l|}{ Other drugs, n (\%) } \\
\hline Metformin & $8236(10.39)$ & $33,375(10.53)$ & .07 \\
\hline Aspirin & $9826(12.40)$ & $41,726(13.16)$ & $<.0001$ \\
\hline Statin & $8336(10.52)$ & $37,395(11.80)$ & $<.0001$ \\
\hline
\end{tabular}

${ }^{\mathrm{a} N} / \mathrm{A}$ : not applicable.

\section{Web-Based System}

We successfully developed a web-based system [19], which contains 2 domains: (1) the drug and cancer page and (2) the overview page. The associations between long-term use of drugs and cancer diseases are shown in AORs with 95\% CIs. The associations are visualized on the overview page, where researchers can compare and contrast the quantified personalized risk of multiple types of cancers for long-term users of the 6 groups of medications at the same time.

\section{The Drug and Cancer Association Page}

Drugs were categorized into 6 groups: (1) antihypertensives; (2) antihyperlipidemics; (3) antidiabetics; (4) antihyperuricemics; (5) NSAIDs; and (6) anxiolytics, hypnotics, and sedatives. As exemplified in Figure 3, the web-based system shows an AOR of 0.830 (95\% CI 0.807-0.853) between statins (3-hydroxy-3-methyl-glutaryl coenzyme A [HMG-CoA] reductase inhibitors) and the overall cancer risk for those aged 20 years or older. Table 3 shows the associations between different drugs and cancers among different age groups gathered from the developed web-based system. 
Figure 3. Display of drug and cancer risk.

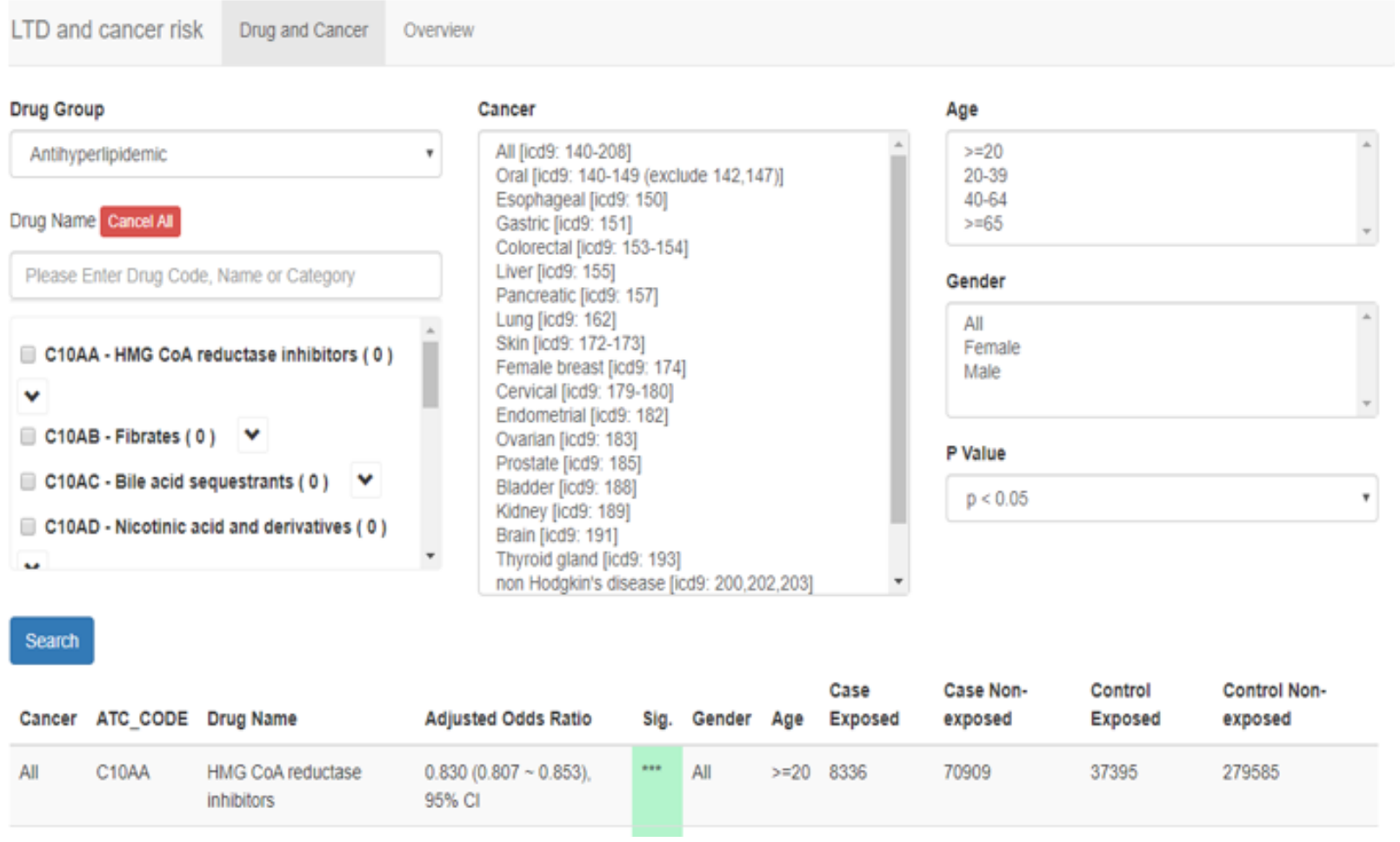


Table 3. Associations between different drugs and cancers among different age groups.

\begin{tabular}{|c|c|c|c|c|c|}
\hline \multirow[t]{2}{*}{ Drug (ATC ${ }^{\text {a }}$ code), cancer type, and age (years) } & \multirow[t]{2}{*}{ Adjusted odd ratio $(95 \% \mathrm{CI})$} & \multicolumn{2}{|c|}{ Case patients, $\mathrm{n}$} & \multicolumn{2}{|c|}{ Control patients, $\mathrm{n}$} \\
\hline & & Exposure & Nonexposure & Exposure & Nonexposure \\
\hline \multicolumn{6}{|l|}{ Aspirin (B01AC06) } \\
\hline \multicolumn{6}{|l|}{ All } \\
\hline$\geq 65$ & $0.954 * *(0.923-0.985)$ & 6964 & 23,485 & 28,665 & 93,131 \\
\hline $40-64$ & $0.871 * * *(0.831-0.913)$ & 2825 & 37,679 & 12,925 & 149,091 \\
\hline $20-39$ & $1.000(0.679-1.472)$ & 37 & 8255 & 136 & 33,032 \\
\hline$\geq 20$ & $0.924 * * *(0.900-0.949)$ & 9826 & 69,419 & 41,726 & 275,254 \\
\hline \multicolumn{6}{|l|}{ Metformin (A10BA02) } \\
\hline \multicolumn{6}{|l|}{ Colorectal } \\
\hline$\geq 65$ & $0.881 *(0.794-0.979)$ & 797 & 4532 & 3231 & 18,085 \\
\hline $40-64$ & $0.799 * * *(0.701-0.912)$ & 456 & 4757 & 2076 & 18,776 \\
\hline 20-39 & $0.448(0.148-1.358)$ & 4 & 776 & 28 & 3092 \\
\hline$\geq 20$ & $0.845 * * *(0.779-0.916)$ & 1257 & 10,065 & 5335 & 39,953 \\
\hline \multicolumn{6}{|l|}{ Sitagliptin (A10BH01) } \\
\hline \multicolumn{6}{|l|}{ Pancreatic } \\
\hline$\geq 65$ & $1.901 *(1.125-3.213)$ & 28 & 632 & 50 & 2590 \\
\hline $40-64$ & $2.303 *(1.109-4.781)$ & 16 & 565 & 27 & 2297 \\
\hline 20-39 & $\mathrm{N} / \mathrm{A}^{\mathrm{b}}$ & 0 & 91 & 0 & 364 \\
\hline$\geq 20$ & $1.981 * *(1.298-3.024)$ & 44 & 1288 & 77 & 5251 \\
\hline \multicolumn{6}{|l|}{ Benzodiazepine derivatives (N05) } \\
\hline \multicolumn{6}{|l|}{ Brain } \\
\hline$\geq 65$ & $1.090(0.787-1.508)$ & 78 & 201 & 256 & 860 \\
\hline $40-64$ & $1.456^{* *}(1.112-1.905)$ & 111 & 379 & 287 & 1673 \\
\hline 20-39 & $2.409 * *(1.364-4.257)$ & 24 & 201 & 46 & 854 \\
\hline$\geq 20$ & $1.379 * *(1.138-1.670)$ & 213 & 781 & 589 & 3387 \\
\hline
\end{tabular}

aTC: Anatomical Therapeutic Chemical.

${ }^{\mathrm{b}} \mathrm{N} / \mathrm{A}$ : not applicable.

$* P<.05$.

$* * P<.01$.

$* * * P<.001$.

\section{The Overview Page}

The web-based system provided an overview of associations between cancers and medications sorted by age, gender, $P$ value, and ATC class of medications (Figure 4). In the cells are AORs of each cancer for different medications, and a confidence interval of $95 \%, 99 \%$, or $99.9 \%$ can be selected by users based on different $P$ values $(P<.05, P<.01, P<.001)$. The web-based system highlights the cells with different colors-from green to white to red-to demonstrate the direction and the extent of these drug-cancer associations. When a user clicks on the Show
Adjusted OR button, the system is able to show all associations. However, if a drug-cancer association pair has a sample size less than 10 patients, the cell stays blank.

Green colors symbolize a significant association between a drug and a cancer with an AOR less than 1. The darker a green color is, the farther from 1 the AOR is. Red colors symbolize a significant association between a drug and a cancer with an AOR greater than 1. The darker a red color is, the farther from 1 the AOR is. White indicates no significant association between a cancer and a drug. 
Figure 4. Display of overview.

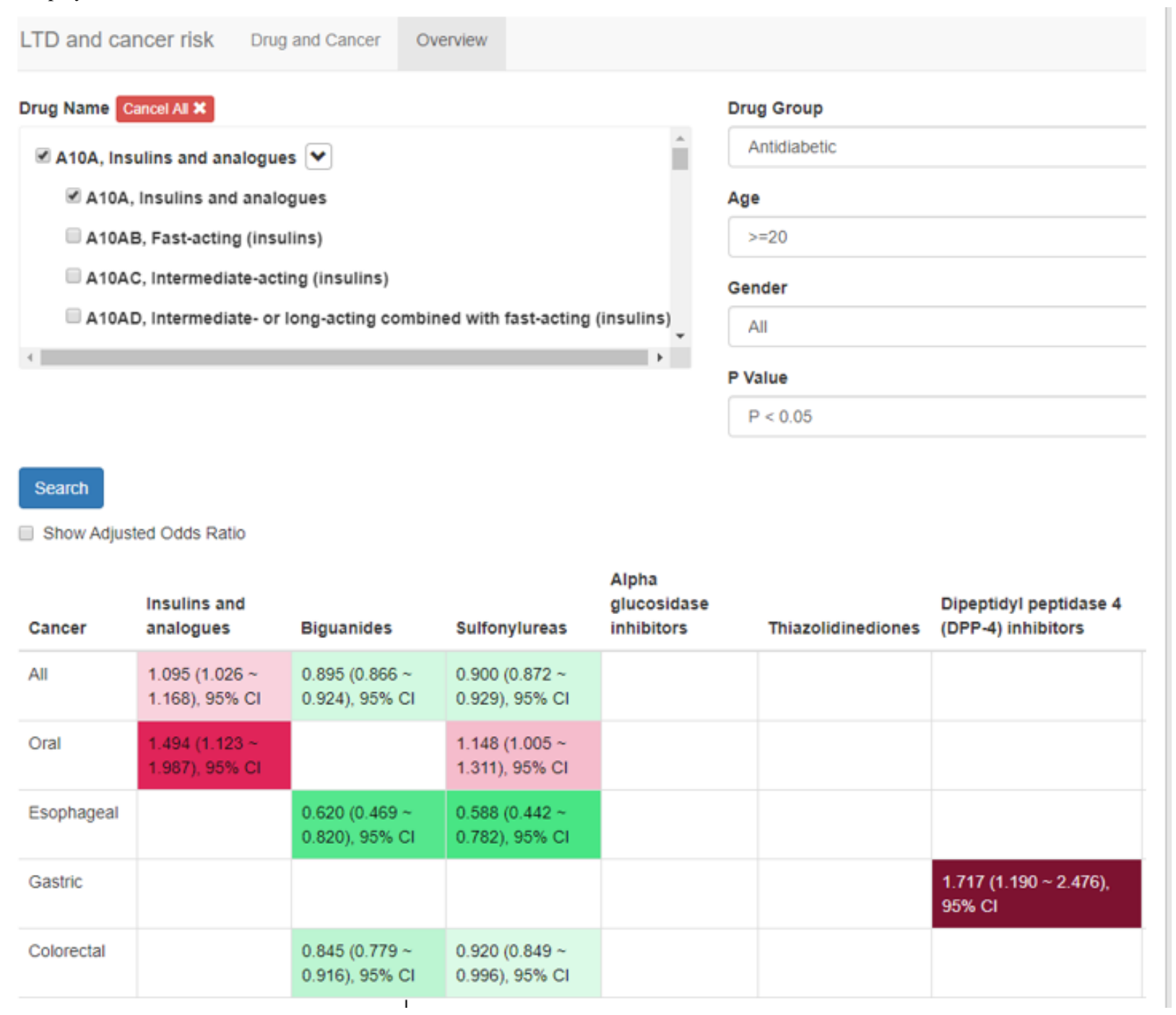

\section{Discussion}

\section{Main Outcomes}

In this nationwide longitudinal retrospective study, we evaluated 79,245 patients with cancer and 316,980 control patients matched by variables including age, sex, and visit date at a 1:4 ratio from the NHIRD in Taiwan, including the follow-up data from 2001 to 2013 of 2 million individuals aged 20 years or older. This web-based system aimed to provide information of medication-cancer associations for users (researchers) to choose potentially clinically relevant ones for further studies (eg, a meta-analysis) and offered a filter by $P$ value. We found aspirin and metformin were significantly associated with reduced cancer risk in those aged 40 to 64 years and 65 years or older, but no significant association was uncovered in those aged 20 to 39 years. A partial explanation for this may lie in the fact that the low prescribing rate or the low cancer incidence among those aged 20 to 39 years rendered it impossible for us to reject the null hypothesis that there were no associations between aspirin and all cancers or between metformin and colorectal cancer.

The long-term use of some drugs was associated with increased risk of certain cancers, such as sitagliptin with pancreatic cancer and benzodiazepines (BZDs) with brain cancer. For example, patients aged 40 to 64 years and 65 years or older treated with sitagliptin had a high risk for pancreatic cancer, but there was not sufficient information for us to estimate such risk among patients aged 20 to 39 years. On the contrary, those aged 20 to 39 years receiving BZDs had a higher risk of brain cancer (AOR $2.409,95 \%$ CI 1.364-4.257; $P=.003$ ) compared with the overall population (AOR 1.379, 95\% CI 1.138-1.670; $P=.001$ ), but there was no significant association between BZDs and brain cancer among those aged 65 years or older.

\section{Biological Mechanisms}

Despite mechanisms between the long-term use of drugs and cancer risk remaining not well understood, our findings were consistent with possible mechanisms proposed in previous studies. Aspirin, metformin, and statins are examples of this. According to previous studies, aspirin reduces prostaglandin generation, which is associated with decreased cellular proliferation, by inhibiting cyclooxygenase isozymes [20]. Metformin activates adenosine monophosphate-activated protein kinase (AMPK), which is a major sensor of whole-body energy metabolism, and activation of AMPK helps to reduce the proliferation of human colon cancer cells [21]. Statins can also decrease intracellular cholesterol production by inhibiting HMG-CoA and may also limit the cellular proliferation required 
for cancer growth [22]. In this study, aspirin, metformin, and statins were found to have significant associations with overall cancer risk, with AORs of 0.924 (95\% CI 0.900-0.949; $P<.001$ ), 0.845 (95\% CI $0.779-0.916 ; P<.001)$, and $0.830(95 \% \mathrm{CI}$ $0.807-0.853 ; P<.001)$.

Additionally, sitagliptin has been suggested to have an association with elevated risk of pancreatitis and pancreatic cancer [23]. In our results, sitagliptin was also significantly associated with pancreatic cancer risk (AOR 1.981, 95\% CI 1.298-3.024; $P=.002$ ). Another intriguing finding in our study was that cancer risk was significantly associated with angiotensin-converting enzyme inhibitors (ACEIs) (AOR 0.854, 95\% CI $0.829-0.880 ; P<.001)$ but not with angiotensin II antagonists (ARBs), even though both ACEIs and ARBs pharmacologically share a similar pathway. A possible explanation is that the stronger inhibitory effect of ACEIs compared with ARBs on angiotensinogen may be associated with cancer risk. However, our system determined associations only, not causalities, between long-term use of medications and risk of cancers. For some of the significant associations, questions about their mechanisms are still left unanswered. Since the results of this study were associations that did not determine the causality, these associations will need further work to confirm mechanisms and causal relationship between long-term use of drugs and cancer risk.

\section{Clinical Implication}

Despite the immense investment in anticancer therapy, cancer remains the leading cause of death globally [24]. Development of an anticancer drug is resource intensive and takes an average of 13 years at a cost up to US \$2.6 billion [25]. The rapidly growing cost and development time have made the pharmaceutical industry a less profitable choice for many investors [26]. Although little attention has been paid to identifying new chemoprevention drugs from existing available drugs, the strategy of using one drug to treat several indications has shown potential success and become an attractive proposition in many areas of medicine, especially in complex disorders [27].

Aspirin is widely used to treat fever and mild pain, but its long-term use may prevent development of squamous cell carcinoma [28], colorectal cancer [29], and hepatocellular carcinoma [30]. Statin use is associated with a reduced risk of pancreatic ductal adenocarcinoma [31] and hepatocellular carcinoma for patients with risk factors [22]. Moreover, metformin, an antidiabetic medication, has drawn attention, since it exhibits an effect on the prevention and treatment of cancers such as colorectal cancer as beneficial as an independent anticancer drug [32]. However, there has been substantial controversy about whether aspirin, statins, and metformin really have anticancer preventive or therapeutic effects on cancers, and often investigations with varying study designs and populations have reached different conclusions. A prudent decision is immediately needed in clinical practices because the use of commonly prescribed medications has been increasing and the resulting burden of cancer can be substantial at the population level [33]. Our study attempted to investigate the magnitude of cancer risk and the benefits of 6 groups of commonly prescribed medications using a large database and appropriate methodology. Our web-based system could potentially show hints of clinical interest for users such as researchers and health care professionals to propose new hypotheses and further undertake research to identify mechanisms or causalities of associations.

\section{Strengths and Limitations}

Strengths of this study include the retrospective study design, long-term follow-up, proper identification of case and control patients, and measurement of the magnitude of association between 6 commonly used groups of medications and cancer risks. Furthermore, confounding factors were appropriately adjusted to reduce the study bias.

We also acknowledge that our research has limitations that need to be addressed. First, drug adherence, self-payment, laboratory data, and lifestyles characteristics such as body mass index, smoking, and family history of cancer were unavailable in the NHIRD. Second, other risk factors for cancer, such as phenotype, genotype, and exposure type, might have influenced the results. Although we applied the match method and adjustment for numerous covariates to control confounding factors, it was impossible to eliminate all confounding factors, particularly indications. Third, all data were collected from the Taiwan NHIRD, and hence, the study population limited the generalization of the results to other countries with different ethnic distribution. Fourth, the results showed associations between the long-term use of drugs and cancer risk but not causation.

Moreover, we did not set a threshold for statistical significance at $0.05 / 45,368 \approx 1.10 \times 10^{-6}$ for multiple testing correction, given the large number of statistical tests and the highly selected patients-patients with cancer and long-term users of medications instead of the general population. Had we set the significance level at $1.10 \times 10^{-6}$, there would not have been enough significant associations to be useful or practical to users. Therefore, we offered in the web-based system a filter by $P$ value, allowing users to choose a $P$ value based on their own need for research. Moreover, considering that there might have been a small number of these highly selected patients, especially after we grouped by drug class, cancer type, age, and gender, we provided users with detailed information of sample sizes on the web-based system, showing the numbers of case and control patients either exposed or not exposed to the study medications.

\section{Conclusion}

This comprehensive retrospective study not only provides an overview of associations of cancer risk with 6 commonly prescribed groups of medications but also helps to narrow the gap in the currently insufficient research on the long-term safety of these medications. With all the quantified data visualized, the system is expected to further facilitate research on cancer risk and prevention. Since our findings have proposed only associations between cancers and long-term use of medications, further clinical trials and meta-analyses are required to assess and confirm their causality. This web-based system could potentially serve as a stepping-stone to exploring and consulting associations between long-term use of drugs and cancer risk. 


\section{Acknowledgments}

This research is sponsored in part by the Ministry of Science and Technology (grant number: MOST 109-2222-E-038-002-MY2), the Ministry of Education (grant number: MOE 109-6604-001-400), and Taipei Medical University (grant number: TMU107-AE1-B18).

\section{Conflicts of Interest}

None declared.

\section{Multimedia Appendix 1}

Supplementary table.

[DOCX File, 17 KB-Multimedia Appendix 1]

\section{References}

1. Diederichs C, Berger K, Bartels DB. The measurement of multiple chronic diseases--a systematic review on existing multimorbidity indices. J Gerontol A Biol Sci Med Sci 2011 Mar;66(3):301-311. [doi: 10.1093/gerona/glq208] [Medline: $\underline{21112963}$

2. Jacobs E, Newton C, Thun M, Gapstur SM. Long-term use of cholesterol-lowering drugs and cancer incidence in a large United States cohort. Cancer Res 2011 Mar 01;71(5):1763-1771 [FREE Full text] [doi: 10.1158/0008-5472.CAN-10-2953] [Medline: 21343395]

3. Suissa S, Hutchinson T, Brophy J, Kezouh A. ACE-inhibitor use and the long-term risk of renal failure in diabetes. Kidney Int 2006 Mar;69(5):913-919 [FREE Full text] [doi: 10.1038/sj.ki.5000159] [Medline: 16518351]

4. Jessen F, Kaduszkiewicz H, Daerr M, Bickel H, Pentzek M, Riedel-Heller S, et al. Anticholinergic drug use and risk for dementia: target for dementia prevention. Eur Arch Psychiatry Clin Neurosci 2010 Nov;260(Suppl 2):S111-S115. [doi: 10.1007/s00406-010-0156-4] [Medline: 20960005]

5. Islam MM, Iqbal U, Walther B, Atique S, Dubey NK, Nguyen P, et al. Benzodiazepine Use and Risk of Dementia in the Elderly Population: A Systematic Review and Meta-Analysis. Neuroepidemiology 2016;47(3-4):181-191 [FREE Full text] [doi: 10.1159/000454881] [Medline: 28013304]

6. Benner J, Glynn R, Mogun H, Neumann P, Weinstein M, Avorn J. Long-term persistence in use of statin therapy in elderly patients. ACC Curr J Rev 2002 Nov;11(6):36. [doi: 10.1016/s1062-1458(02)00914-5]

7. Moysich KB, Beehler GP, Zirpoli G, Choi J, Baker JA. Use of Common Medications and Breast Cancer Risk. Cancer Epidemiol Biomarkers Prev 2008 Jul 15;17(7):1564-1595. [doi: 10.1158/1055-9965.epi-07-2828]

8. Karlstad O, Starup-Linde J, Vestergaard P, Hjellvik V, Bazelier M, Schmidt M, et al. Use of insulin and insulin analogs and risk of cancer - systematic review and meta-analysis of observational studies. Curr Drug Saf 2013 Nov;8(5):333-348 [FREE Full text] [doi: 10.2174/15680266113136660067] [Medline: 24215311]

9. Singh S, Singh H, Singh PP, Murad MH, Limburg PJ. Antidiabetic Medications and the Risk of Colorectal Cancer in Patients with Diabetes Mellitus: A Systematic Review and Meta-analysis. Cancer Epidemiol Biomarkers Prev 2013 Sep 16;22(12):2258-2268. [doi: 10.1158/1055-9965.epi-13-0429]

10. Gandini S, Palli D, Spadola G, Bendinelli B, Cocorocchio E, Stanganelli I, et al. Anti-hypertensive drugs and skin cancer risk: a review of the literature and meta-analysis. Crit Rev Oncol Hematol 2018 Feb;122:1-9. [doi: 10.1016/j.critrevonc.2017.12.003] [Medline: 29458778]

11. Ni H, Rui Q, Zhu X, Yu Z, Gao R, Liu H. Antihypertensive drug use and breast cancer risk: a meta-analysis of observational studies. Oncotarget 2017 Sep 22;8(37):62545-62560 [FREE Full text] [doi: 10.18632/oncotarget.19117] [Medline: 28977968]

12. Ioannidis J, Zhou Y, Chang C, Schully S, Khoury M, Freedman A. Potential increased risk of cancer from commonly used medications: an umbrella review of meta-analyses. Ann Oncol 2014 Jan;25(1):16-23 [FREE Full text] [doi:

10.1093/annonc/mdt372] [Medline: 24310915]

13. Raghupathi W, Raghupathi V. Big data analytics in healthcare: promise and potential. Health Inf Sci Syst 2014;2:3 [FREE Full text] [doi: 10.1186/2047-2501-2-3] [Medline: 25825667]

14. Wang YH, Nguyen PA, Islam MM, Li YC, Yang HC. Development of Deep Learning Algorithm for Detection of Colorectal Cancer in EHR Data. 2019 Presented at: 17th World Congress on Medical and Health Informatics (MEDINFO 2019); Aug 25-30, 2019; Lyon, France.

15. Deyo RA, Cherkin DC, Ciol MA. Adapting a clinical comorbidity index for use with ICD-9-CM administrative databases. J Clin Epidemiol 1992 Jun;45(6):613-619. [doi: 10.1016/0895-4356(92)90133-8] [Medline: 1607900]

16. Rosner B. Fundamentals of Biostatistics. Toronto, ON, Canada: Nelson Education; 2015.

17. Choi N, Park B, Jeong S, Yu K, Yoon B. Nonaspirin Nonsteroidal Anti-inflammatory Drugs and Hemorrhagic Stroke Risk. Stroke 2008 Mar;39(3):845-849. [doi: 10.1161/strokeaha.107.497040] 
18. Thrift AG, McNeil JJ, Forbes A, Donnan GA. Risk of primary intracerebral haemorrhage associated with aspirin and non-steroidal anti-inflammatory drugs: case-control study. BMJ 1999 Mar 20;318(7186):759-764 [FREE Full text] [doi: 10.1136/bmj.318.7186.759] [Medline: 10082697 ]

19. LTD and cancer risk. Taiwan Artificial Intelligence for Medicine \& Health Innovations Center. URL: http://ltd-cancer. aimhi.tw/ [accessed 2021-02-09]

20. Sostres C, Gargallo CJ, Lanas A. Aspirin, cyclooxygenase inhibition and colorectal cancer. World J Gastrointest Pharmacol Ther 2014 Feb 06;5(1):40-49 [FREE Full text] [doi: 10.4292/wjgpt.v5.i1.40] [Medline: 24605250]

21. Higurashi T, Hosono K, Takahashi H, Komiya Y, Umezawa S, Sakai E, et al. Metformin for chemoprevention of metachronous colorectal adenoma or polyps in post-polypectomy patients without diabetes: a multicentre double-blind, placebo-controlled, randomised phase 3 trial. Lancet Oncol 2016 Apr;17(4):475-483. [doi: 10.1016/s1470-2045(15)00565-3]

22. Singh S, Singh PP, Singh AG, Murad MH, Sanchez W. Statins are associated with a reduced risk of hepatocellular cancer: a systematic review and meta-analysis. Gastroenterology 2013 Feb;144(2):323-332. [doi: 10.1053/j.gastro.2012.10.005] [Medline: 23063971]

23. Elashoff M, Matveyenko AV, Gier B, Elashoff R, Butler PC. Pancreatitis, pancreatic, and thyroid cancer with glucagon-like peptide-1-based therapies. Gastroenterology 2011 Jul;141(1):150-156 [FREE Full text] [doi: 10.1053/j.gastro.2011.02.018] [Medline: 21334333]

24. Heron M, Anderson RN. Changes in the Leading Cause of Death: Recent Patterns in Heart Disease and Cancer Mortality. NCHS Data Brief 2016 Aug;254:1-8 [FREE Full text] [Medline: 27598767]

25. Avorn J. The $\$ 2.6$ billion pill--methodologic and policy considerations. N Engl J Med 2015 May 14;372(20):1877-1879. [doi: 10.1056/NEJMp1500848] [Medline: 25970049]

26. Pushpakom S, Iorio F, Eyers PA, Escott KJ, Hopper S, Wells A, et al. Drug repurposing: progress, challenges and recommendations. Nat Rev Drug Discov 2019 Jan;18(1):41-58. [doi: 10.1038/nrd.2018.168] [Medline: 30310233]

27. Hopkins AL. Drug discovery: Predicting promiscuity. Nature 2009 Nov 12;462(7270):167-168. [doi: 10.1038/462167a] [Medline: 19907483]

28. Pandeya N, Olsen C, Thompson B, Dusingize J, Neale R, Green A, QSkin Study. Aspirin and nonsteroidal anti-inflammatory drug use and keratinocyte cancers: a large population-based cohort study of skin cancer in Australia. Br J Dermatol 2019 Oct;181(4):749-760. [doi: 10.1111/bjd.17938] [Medline: 30920641]

29. García Rodríguez LA, Soriano-Gabarró M, Bromley S, Lanas A, Cea Soriano L. New use of low-dose aspirin and risk of colorectal cancer by stage at diagnosis: a nested case-control study in UK general practice. BMC Cancer 2017 Sep 07;17(1):637 [FREE Full text] [doi: 10.1186/s12885-017-3594-9] [Medline: 28882113]

30. Simon TG, Ma Y, Ludvigsson JF, Chong DQ, Giovannucci EL, Fuchs CS, et al. Association Between Aspirin Use and Risk of Hepatocellular Carcinoma. JAMA Oncol 2018 Dec 01;4(12):1683-1690 [FREE Full text] [doi: 10.1001/jamaoncol.2018.4154] [Medline: $\underline{\text { 30286235] }}$

31. Choi JH, Lee SH, Huh G, Chun JW, You MS, Paik WH, et al. The association between use of statin or aspirin and pancreatic ductal adenocarcinoma: A nested case-control study in a Korean nationwide cohort. Cancer Med 2019 Dec;8(17):7419-7430 [FREE Full text] [doi: $\underline{10.1002 / \mathrm{cam} 4.2617]}$ [Medline: $\underline{\text { 31637875] }}$

32. Kamarudin MNA, Sarker MMR, Zhou J, Parhar I. Metformin in colorectal cancer: molecular mechanism, preclinical and clinical aspects. J Exp Clin Cancer Res 2019 Dec 12;38(1):491 [FREE Full text] [doi: 10.1186/s13046-019-1495-2] [Medline: $\underline{31831021]}$

33. Ioannidis J, Zhou Y, Chang C, Schully S, Khoury M, Freedman A. Potential increased risk of cancer from commonly used medications: an umbrella review of meta-analyses. Ann Oncol 2014 Jan;25(1):16-23 [FREE Full text] [doi:

10.1093/annonc/mdt372] [Medline: 24310915]
Abbreviations
ACEI: angiotensin-converting enzyme inhibitors
AMPK: adenosine monophosphate-activated protein kinase
AOR: adjusted odds ratio
ARB: angiotensin II antagonist
ATC: Anatomical Therapeutic Chemical
BZD: benzodiazepine
HMG-CoA: 3-hydroxy-3-methyl-glutaryl coenzyme A
ICD-9-CM: International Classification of Disease, Ninth Revision, Clinical Modification
NHI: National Health Insurance
NHIRD: National Health Insurance Research Database
NSAID: nonsteroidal anti-inflammatory drug
PHP: Hypertext Preprocessor 
Edited by G Eysenbach; submitted 13.06.20; peer-reviewed by YL Cheong, E Omaki; comments to author 22.09.20; revised version received 29.09.20; accepted 17.01.21; published 15.02.21

Please cite as:

Yang HC, Islam MM, Nguyen PAA, Wang CH, Poly TN, Huang CW, Li YCJ

Development of a Web-Based System for Exploring Cancer Risk With Long-term Use of Drugs: Logistic Regression Approach JMIR Public Health Surveill 2021;7(2):e21401

URL: http://publichealth.jmir.org/2021/2/e21401/

doi: 10.2196/21401

PMID: $\underline{3587043}$

CHsuan-Chia Yang, Md Mohaimenul Islam, Phung Anh Alex Nguyen, Ching-Huan Wang, Tahmina Nasrin Poly, Chih-Wei Huang, Yu-Chuan Jack Li. Originally published in JMIR Public Health and Surveillance (http://publichealth.jmir.org), 15.02.2021. This is an open-access article distributed under the terms of the Creative Commons Attribution License (https://creativecommons.org/licenses/by/4.0/), which permits unrestricted use, distribution, and reproduction in any medium, provided the original work, first published in JMIR Public Health and Surveillance, is properly cited. The complete bibliographic information, a link to the original publication on http://publichealth.jmir.org, as well as this copyright and license information must be included. 